\title{
Feasibility and Acceptance of a Novel Nutrition and Exercise Intervention to Manage Excess Gestational Weight Gain: Focus group study in Ontario, Canada
}

\section{Rishma Walji ${ }^{1}$, Olive Wahoush ${ }^{2}$ and Stephanie A Atkinson ${ }^{3 *}$}

${ }^{1}$ Post-doctoral Fellow, Faculty of Health Sciences, McMaster University, 1280 Main Street West, Hamilton, ON, Canada

${ }^{2}$ Assistant Dean, Undergraduate Nursing Programs, School of Nursing, Faculty of Health Sciences, McMaster University, 1280 Main Street West, Hamilton, ON, Canada ${ }^{3}$ Professor and Associate Chair Research, Department of Pediatrics, Faculty of Health Sciences, McMaster University, 1280 Main Street West, Hamilton, ON, Canada

\begin{abstract}
In preparation for designing a randomized clinical trial aimed to manage gestational weight gain during pregnancy, we conducted a qualitative study using focus groups of women and health care providers for the purpose of identifying the enablers or barriers that support or limit nutrition and exercise modifications for women during pregnancy and to determine the feasibility of such a program in the community. A purposeful sample of pregnant or recently pregnant (within the last year) women $(n=21)$ and health care providers $(n=7)$ were recruited from community health clinics in Hamilton, Canada. A semi-structured focus group guide was tailored to each target group. Qualitative analysis was completed using NVivo 9 software and key conceptual categories of behavioural, control and normative beliefs were identified from transcribed interview texts and field notes. The results indicated that to make nutrition and exercise interventions more accessible to women of various backgrounds, key facilitators should include: behavioural beliefs of the importance of nutrition and exercise in pregnancy, family support and nutritional education. Identified barriers included: communication with healthcare providers, inter-professional collaboration, language, and lack of specific instructions in currently available guidelines. Collectively, information from women and health providers enabled a clear understanding of barriers, enablers and opportunities for the successful implementation of the planned nutrition and exercise intervention for gestational weight gain management. The identified factors were used to design a clinical randomized trial of a structured and monitored nutrition and exercise program for women beginning in early pregnancy.
\end{abstract}

Keywords: Pregnancy; Exercise; Nutrition; Gestational weight gain; Planned behaviour theory; Focus group

\section{Introduction}

Women who enter pregnancy overweight or obese and those who gain excessive weight during pregnancy represent a clinical challenge that impacts on adverse pregnancy and birth outcomes. Alarmingly, the proportion of Canadian women of child-bearing age (18 to 39 years) who were overweight or obese rose from $34 \%$ in 1978 [1] to $53 \%$ in 2007-2009 [2]. Further, excess gestational weight gain when compared to the recent Institute of Medicine guidelines for target weight gain in pregnancy [3] is documented in 55-75\% of Canadian women who enter pregnancy overweight or obese, and up to $40 \%$ of women who enter pregnancy within normal weight categories of Body Mass Index (BMI) $[4,5]$. Overweight on entering pregnancy and excess Gestational Weight Gain (GWG) are independent major risk factors for both maternal and infant complications such as gestational diabetes, preeclampsia, hypertension, macrosomia, shoulder dystocia and more [6,7]. Yet, despite the enormous potential for lifestyle changes to reduce morbidity and mortality and the ever-expanding research on perinatal health, the efficacy of nutrition and exercise counseling in primary maternity care remains poorly understood by health care providers.

While it is assumed that adopting a healthier diet and increasing physical activity in pregnancy can modify weight gain, systematic reviews of such intervention trials as described below have yielded mixed results [8-10]. Despite nutrition and/or exercise being identified in meta-analysis of existing trials as effective in the management of GWG [8-10], the magnitude of the effect on GWG and the overall conclusions of these reviews are inconsistent. The latter is attributed to the poor quality of the published studies, the effect of combining randomized and single-arm intervention trials, significant heterogeneity among the studies such as differences in the weight categories of women included (overweight/obese alone versus mixed weight categories) and interventions employed, and lack of well prescribed "active ingredients or characteristics" of the diet and exercise interventions [8-10] Further, none of the systematic reviews was able to identify direct links between intervention type and study outcomes nor specific behavior change strategies that were effective. In addition, interventions have not been proven to be feasible, effective and acceptable to women in the community, especially those identified as vulnerable. This research gap limits development of evidence-based guidelines for lifestyle interventions to prevent excessive GWG and associated adverse outcomes-clinical problems widely identified as public health priorities [10]. To address these gaps in knowledge, we designed a randomized clinical trial that will examine a specific nutrition plan and a structured and monitored physical activity plan in pregnant women beginning by the end of the first trimester of pregnancy (NCT01689961). Prior to implementing this trial, we conducted a focus group study to inform selection of intervention components and tools to measure nutrition and exercise practices as well as to understand the recruitment and retention challenges in conducting the randomized trial within a sociodemographically diverse population of women.

The specific aim of the reported study in pregnant/recently pregnant women (within one year of delivery) and health providers was to identify the enablers and barriers that support or limit nutrition

*Corresponding author: Dr. Stephanie Atkinson, Department of Pediatrics McMaster University, 1280 Main Street West, Hamilton, ON, Canada, Tel: 905-521-2100; Fax: 905-308-7548; E-mail: satkins@mcmaster.ca

Received April 03, 2013; Accepted May 25, 2013; Published May 28, 2013

Citation: Walji R, Wahoush O, Atkinson SA (2013) Feasibility and Acceptance of a Novel Nutrition and Exercise Intervention to Manage Excess Gestational Weight Gain: Focus group study in Ontario, Canada. Primary Health Care 3: 134 doi:10.4172/2167-1079.1000134

Copyright: (c) 2013 Walji R, et al. This is an open-access article distributed under the terms of the Creative Commons Attribution License, which permits unrestricted use, distribution, and reproduction in any medium, provided the original author and source are credited. 
and exercise modification for women to effectively manage their GWG during pregnancy. This information was utilized to inform the feasibility and design of the clinical randomized trial of a novel structured and monitored nutrition and exercise intervention in pregnant women.

\section{Theoretical Framework}

The theory of planned behaviour guided the development of our focus group questions and data analysis. In brief, the theory of planned behaviour suggests that a person's behaviour is determined by intention to perform the behaviour and that this intention is, in turn, a function of attitude toward the behaviour (desirability) and subjective norms (valued by significant others) [11]. The best predictor of behaviour is intention. Intention is the cognitive representation of a person's readiness to perform a given behaviour, and it is considered to be the immediate antecedent of behaviour. The Theory of Planned Behaviour suggests that performance of a given behaviour is a combination of intentions and perceived behavioural control [12]. This approach stipulates that intentions predict behaviour, and that three sets of beliefs mediate behavioural intentions in relation to the following criteria: (i) behavioural beliefs, i.e., attitudes based on perceived benefits and risks, (ii) control beliefs, i.e., perceptions relating to access and control over necessary resources and opportunities; and (iii) normative beliefs, i.e., social influences, determined by perceptions of the views of others.

According to this theory, favourable behavioural, control, and normative beliefs related to nutrition and physical activity of pregnant women would likely be associated with their intentions and subsequent levels of physical activity or diet behaviours. If a pregnant woman believes that maintaining control of weight gain is important for her health and the health of her newborn she will be more likely to attend to diet and exercise. However, if a pregnant woman perceives exercise is potentially harmful or that weight gain is not able to be controlled because of hormonal influences in pregnancy, then her beliefs about controlling weight gain are likely diminished.

This study was primarily concerned with women's lifestyle behaviours related to nutrition and exercise experiences in pregnancy. Health care providers are generally considered key resources and have the potential to influence their patient/client behaviours in relation to motivation as well as education about healthy lifestyles. While health care providers can serve as access points for important information about the context of weight management in pregnancy care, the actual practice of educating of women about weight gain in pregnancy is documented to be infrequent and many barriers exist [13-15]. For these reasons, health care providers were also asked for information about their current practices as well as perceptions of barriers and challenges to managing gestational weight gain.

\section{Materials and Methods}

This qualitative study was completed in community health centres providing care to pregnant women within the Hamilton area. Care in these clinics is provided by medical doctors, nurses, and dieticians.

Hamilton was the site selected for this focus group study as this will be one of the sites for the randomized trial. Hamilton is the third largest city in Ontario and has an economically and ethno-racially diverse population. Primary health care for pregnant women is provided by a full range of health service provider agencies and two large hospital corporations. Primary health service agencies include, community health centres, group practices of family physicians, family health teams, nurse practitioner led clinics and midwifery practices. Public Health programs are also available for prenatal education for women.

A purposive sampling approach was used to recruit women who were either currently pregnant or had been pregnant in the past year. The aim was to include participants typical of those who would participate in our planned randomized clinical trial, and who could speak to the topic of lifestyle habits in pregnancy. The goal in this case is not to sample random or representative distributions of a particular population, but to collect rich information about gestational weight gain which requires information from participants who have experience with pregnancy [16].

Participants were recruited via poster advertising in primary care clinics and public health settings. We conducted four focus groups, two with health care providers and 2 with pregnant or recently pregnant women. Recently pregnant women were all less than 1 year postpartum and were included in the study so that they could comment on their experiences with weight management throughout their recent pregnancy. Participants included persons of different ethnic and cultural backgrounds and socioeconomic status, as well as women of varying weight, parity and a wide range of experience and knowledge in healthy lifestyles. The range of health care providers also represented different experiences from working in health professional settings across the city as well as having different professional training experiences and roles in pregnancy care.

Focus groups loosely followed a discussion guide (Table 1), and also allowed for organic group discussion. Focus group questions were

\begin{tabular}{|c|c|}
\hline Women & Health care providers \\
\hline $\begin{array}{l}\text { First please tell us your thoughts about weight gain in pregnancy } \\
\text { a. what you know } \\
\text { b. } \quad \text { or have experienced. }\end{array}$ & $\begin{array}{l}\text { First please tell us your thoughts about weight gain in pregnancy } \\
\text { a. (probe) what you have seen in practice }\end{array}$ \\
\hline $\begin{array}{l}\text { We want to know about your experiences with weight gain during or after } \\
\text { pregnancy, tell us if you tried anything to manage your weight and why you tried } \\
\text { that or if you did not try anything. } \\
\begin{array}{ll}\text { a. Helpful factors } \\
\text { b. Barriers } \\
\text { c. } \quad \text { Challenges about weight gain or control }\end{array}\end{array}$ & $\begin{array}{l}\text { What are some issues you have dealt with when trying to help a woman achieve } \\
\text { appropriate weight gain during pregnancy } \\
\text { a. What helps compliance with your recommendations? } \\
\text { b. What are your resources for good information (both for patient and for } \\
\text { the health care provider) } \\
\text { c. What are the barriers to achieving optimal weight gain }\end{array}$ \\
\hline $\begin{array}{l}\text { What do you think would be helpful for you or other women like you to manage } \\
\text { their weight during and after pregnancy? }\end{array}$ & $\begin{array}{l}\text { What do you think would be helpful for women to manage their weight during } \\
\text { and after pregnancy? }\end{array}$ \\
\hline $\begin{array}{l}\text { Our planned research is ... } \\
\text { What do you think about what we are asking women to do? } \\
\text { a. Would you be able to do what is being asked? } \\
\text { b. What are some things that might make it easier? } \\
\text { c. What are some challenges? }\end{array}$ & $\begin{array}{l}\text { Our planned intervention is ... } \\
\text { a. What do you think of this interventions' feasibly } \\
\text { b. } \quad \text { Do you think this intervention would be effective? Why or why not? }\end{array}$ \\
\hline Do you have any additional comments that we have not asked? & Do you have any additional relevant comments that we have not asked? \\
\hline
\end{tabular}

Table 1: Discussion guide for focus groups (the sub-questions were used as probes to elicit discussion). 
tailored to the population group. Health providers were asked to discuss both the role they believe nutrition plays in maternal-fetal health and their approach to nutrition, exercise, and weight gain counselling during the prenatal period. The women were asked about their experiences during and after pregnancy with respect to nutrition, exercise and weight gain. In addition, we discussed the planned intervention for our future randomized clinical trial (NCT01689961) and asked both the health care providers and women to comment on their thoughts and preferences related to the interventions. All narratives were transcribed verbatim and then content analyzed by the authors to produce an initial coding system based on common concepts and commonly recurring themes.

Qualitative analysis was completed using NVivo 9 software to facilitate the identification and coding of themes from interviews to help identify and mark the location of key conceptual categories in transcribed interview texts and field notes [17]. Two authors coded the transcripts (RW, OW), one of whom was also present at the focus groups (RW). Key themes were identified and coded based on emergent ideas. Text was organized into tables that mapped participant responses to each of the focus group questions and to our study questions, a process which helped shape our interpretations. Theoretical or concept saturation was reached when gathering more data about a category yielded no further insights [18]. Credibility of this study was achieved by recruiting women who were pregnant or recently pregnant and using open questions that asked women about gestational weight gain, their experiences and thoughts about it without directing participants to provide a specific type of response. Conformability was promoted by a repeat analysis of transcripts after a one week time period and each author completed the preliminary analysis independently. Results were then checked for congruence of concepts and themes. No amendments or changes were necessary.

Ethical approval was obtained from the Research Ethics Board of Hamilton Health Sciences/McMaster University and each participant signed a consent form prior to the focus group session.

\section{Results}

The 21 women included 12 who were currently pregnant and nine who had been pregnant within the past year. There were two focus groups of women, and both groups consisted of pregnant and recently pregnant women. The mean parity of 18 of the women (three declined providing this information) was 1.4 (range 0-5). English was a second language for $47.6 \%$ of the women. The seven health care providers consisted of one nurse, one general practitioner, two first year medical residents, two second year medical residents and one dietician. The health care providers' and women's perspectives of factors affecting a pregnant woman's ability to achieve an intended behaviour are presented separately as each group identified different factors.

The findings are presented according to the analytical typologies: (i) behavioural beliefs, (ii) control beliefs and (iii) normative beliefs. Verbatim quotes from the study participants are labelled by type of participant and by Focus Group (FG) number. Taking into account the information gathered within the context of each of the belief categories, we developed two models depicting the barriers and promoters of adherence to diet and exercise in pregnancy according to the theory of planned behaviour. The themes of information collected from women to one of the three types of belief categories featured in the model are displayed in Figure 1. For example, women in this study identified financial issues and family life factors that impacted their beliefs about their ability to control diet and exercise behaviour. Comments from health care providers were grouped into one of the three belief categories (Figure 2). Providers related lack of time or lack of nutrition knowledge as factors that affected their beliefs about how they might help women manage weight gain in pregnancy which in turn impacted their intentions for prenatal visits.

\section{Women's responses}

Beliefs about nutrition and leading a healthy lifestyle: Attitude toward behaviour (healthy lifestyle, nutrition and exercise behaviour) is defined as the individual's positive or negative feelings about performing that behaviour. It is determined through an assessment of one's beliefs regarding the consequences arising from behaviour and an evaluation of the desirability of these consequences.

In the current focus groups, women generally agreed that nutrition and exercise were important to maintaining a healthy pregnancy and managing gestational weight gain. The benefits of health for baby and family were strong, even over other potential obstacles, such as price and convenience.

I'm always out, so I eat out maybe too much. But I try to pick places where they have vegetables. For the kids and that, even though it's expensive. (Woman FG 1)

Other women commented about their main source of information about nutrition, for example, prenatal classes.

I went to a prenatal class that told us a lot about what to eat, what not to eat, and stuff like that. (Woman FG 2)

During each focus group, a few pregnant women and mothers indicated that they were interested in how they might measure their progress in exercise. For example,

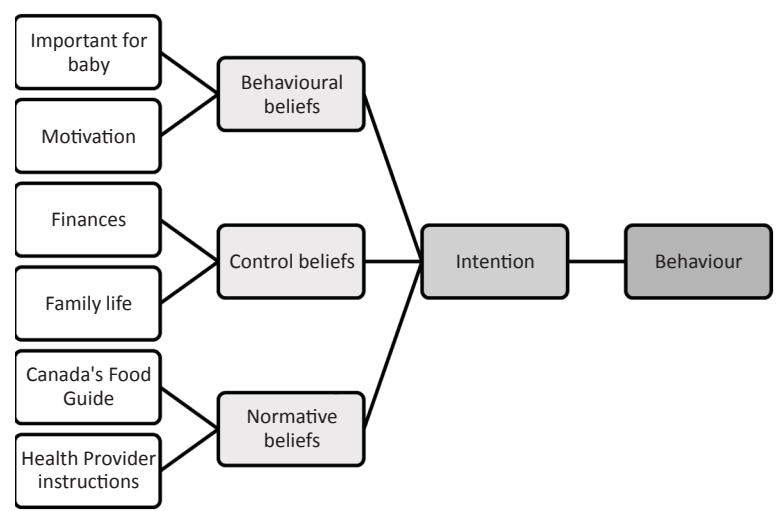

Figure 1: Behavioural model for women adapted from Ajzen's Theory of Planned Behaviour [11].

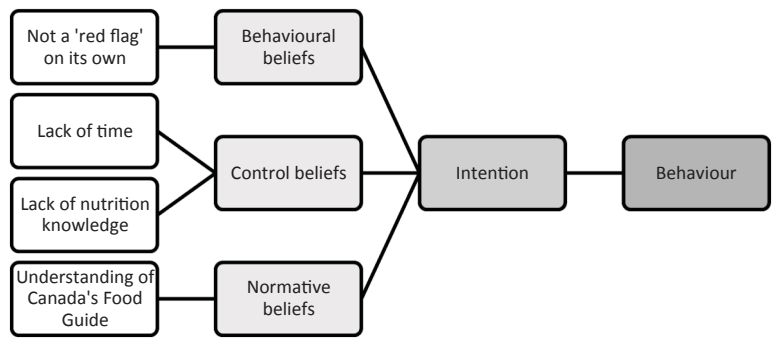

Figure 2: Behavioural model for health care providers adapted from Ajzen's Theory of Planned Behaviour [11]. 


\section{I'd be interested to see how many steps I take a day [related to exercise]} (Women FG 1)

Beliefs about their ability to make change: Behavioural control is defined as one's perception of the difficulty of making nutritional or lifestyle modifications. The theory of planned behaviour views the control that people have over their behaviour as lying on a continuum from behaviours that are easily performed to those requiring considerable effort and resources.

Behavioural control can include both actual control and perceived control. Actual barriers for women such as finances were a major discussion point with the women from these community sites.

Unless I come here [to the community centre], the most vegetables I get is coming here. It's hard. Financially, it's difficult. Because I said I spend $\$ 40$ alone just on drinks. So that was chocolate milk, regular milk, a couple boxes of juices and about 5 bottles of water. Have you seen the food guide? Realistically, [what] these kids and I being pregnant should be eating. It's very difficult to say follow the food guide, you know that a family size like us with 7 , it's not realistic. (Women FG 1)

Demands of family life were also mentioned as a limitation or barrier to physical exercise. Comments in this regard included:

I think because being with [my other] children, I try to exercise but it won't work. It's not working. (Woman FG 1)

When I was pregnant with [my first child], when I was hungry, I would eat. But now when I want some food, [my first child] wants me to play with her so I have to suffer, starving. (Woman FG1)

Beliefs about societal expectations regarding nutrition and healthy lifestyle: A subjective norm is defined as an individual's perception of whether people important to the individual think the behaviour should be performed. The contribution of the opinion of any given factor is weighted by the motivation that an individual has to comply with the person of influence.

Women generally agreed that societal norms favour optimal weight and healthy eating during pregnancy. In the women's group, those who did not attempt healthy eating or exercise were at first reluctant to talk about their experiences in the group until reassured that others had similar experiences. Women reported that accepted dietary behaviours are detailed in Health Canada's food guide [19] and that was the resource that most women and health care providers referred to. Despite the health care providers and women both acknowledging that they did not typically discuss lifestyle details with each other, women were aware of the societal norms that eating healthy and exercise is widely accepted and encouraged.

And the cost of food is really expensive, but again, the serving requirements are so, a lot, like if you watch $\mathrm{Dr}$. Oz and stuff, they show you. Like a big bowl in order for you to get, you know, an adequate amount. So that's why they say, you know, like take a vitamin or something so it's packed into one pill. Because the average person can't really consume that. (WomenFG1)

Women referred to expectations from their health care providers and that acceptance by their health care providers was important.

But I'm in the high-risk category. But every time I went, and they took the blood pressure and everything, it was always normal. So they were happy with that. Like I had no complications with being overweight at all. My sugar test was good, my diabetes was negative, so they were quite happy. (Women FG 2)
Normative beliefs of the women were associated with advice and instructions they received from their doctors.

Well they told me to avoid like a lot of fat food, which I didn't do a very good job of, but they told me avoid a lot of, you know, eating fast foods and eat healthier and stuff like that. Even though I had trouble doing that, I was still able to stay in the weight gain that they wanted me to. And I think part of that is because I did a lot of exercise like walking and swimming and things like that. (Woman FG2)

I had a lot of weight gain in my first pregnancy. I don't know if that's the reason I push for more than 2 hours but now I'm being more careful. The first time I gained 50 pounds and [the doctors] told me it would make my delivery more difficult. So now I'm eating healthy and getting more exercise. Less fat, and I try cereal and other foods. And veggies. And less fruit because of the sugar. (Woman FG 2)

\section{Health care providers' responses}

Beliefs about weight gain, nutrition and leading a healthy lifestyle: The health care providers reported calculating the Body Mass Index (BMI) of pregnant women at their first prenatal visit and based on the calculated BMI advised them how much or how little weight to gain using guidelines from Health Canada (base on the IOM guidelines [3]) or the Society for Obstetrics and Gynecology of Canada [20]. Women are then generally weighed at every follow up visit but further counselling on GWG is not routine unless there's a "red flag".

The belief about healthy lifestyle is shaped not only by an individual's beliefs but also what is accepted by society and health care providers. Health care providers might influence a woman's belief about a certain intervention or healthy behaviour.

Health care providers were supportive of positive nutrition and exercise interventions and indicated that not only the planned intervention but also the use of an incentive such as the pedometer would benefit women. In their own practice, however, the risk associated with excess gestational weight gain was not as prominent in practice as we had expected and was only discussed if a 'red flag' occurred (such as the woman being at risk for hypertension or diabetes).

Health care providers in this study thought that enabling prompt feedback to women might be helpful and promote walking. For example, in response to our question about using an electronic device to measure walking we heard that, [pedometer] People like things, they like numbers and things and gadgets and like I say it might be a bit empowering cause I think in some cases people think there's so much against them [obstacles] that just might be a positive to say look at how many steps you're doing and there's a number and you wear it and you get things. (HCP FG2)

Beliefs about their ability to make change: In the case of health care providers, control beliefs they cited were lack of time and lack of detailed clinical knowledge. The fact that there are competing priorities for health assessment in a visit meant that women were not typically discussing their weight issues with their physician.

Again, you know because of lack of time.., also because of the way our clinic is set up, we don't always see the same patient so teachings include multiple care providers. It's hard to follow-up, that you actually go and get this or that and the other. I think its hard with diet because with anything else there is something you can measure; it's easier, for example a $C B C$, but with this its difficult because it's hard to measure if you have been taking enough protein or whatever cause there is no actual measurement tool so the onus is on the patient. (HCP FG 1)

Knowledge gaps may further limit the likelihood of discussing diet 
with women; in addition, health checks during pregnancy are already detailed with planned tests for each planned visit during the pregnancy.

I would certainly agree to the fact that lack of total knowledge because I can't calculate the protein and stuff and then tell the patient with confidence. And lack of time for sure I mean the first two appointments are so full of so many screenings and this and that. (HCP FG 2)

Health care providers also shared information about their competing priorities in their practice and their need for tools that help them provide quality information at the relevant time. This may be especially important for those working in settings where women have multiple needs.

I would agree to competing priorities to the provider as well as the client and especially life is sort of chaotic [diet and exercise counselling] sort of falls to the back burner. And as well as my own knowledge base and I was just thinking it would be good when talking about resources to have some sort of enabler where it says "say this" or this is the 30 second or 60 second [instructions] for the health provider, as well to say because you know we especially myself and primary care out in the shelters, I rely on algorithms a lot, it's sort of what to do at each visit so it's something for the providers as well that would be probably very useful. (HCP FG2)

Beliefs about societal expectations regarding nutrition and healthy lifestyle: Health care providers tended to rely on the women's concerns. Diet and exercise were not topics discussed in detail unless the woman was obese or if the woman was inquiring about it herself.

If they are concerned about it then you just kind of reassure them, tell them, advise them to eat healthy and to advise them about the food they should be eating more than focusing on the weight. But at the same time if you can perceive it as being a problem in the future, that they might be you know a little too lax, you might bring up the guidelines and show them what they should be gaining at each stage of the pregnancy. (HCP FG 1)

The health care providers tended to give their advice based on red flags that are necessary to identify in medicine but also relied on the women's concerns. Diet was not a topic discussed in detail unless the woman was obese or if the woman was inquiring about it herself. In terms of advice, the only resource identified was Eating Well with Canada's Food Guide [19], but they did not give additional guidance on how to cook meals or specific nutrients that were needed. The latter type of guidance was seen as the role of the dietician, but the primary care practitioners only made referrals to a dietitian in cases of serious health concerns identified in the mothers.

Certainly if you have any extremely large baby it puts you at higher risk for shoulder dystocia during the delivery which causes all sorts of problems with the baby. So it's definitely something that we ....consider. I would say that [diet] is probably something that we don't have as much time and don't spend as much time talking about with pregnant women because you're expecting them to gain weight. And certainly if you see huge gains it raises a red flag, but it's definitely not the focus of the visit (group members make agreeing noises). So it's certainly something that could get missed or not addressed as in depth as other issues might be. (HCP, FG1)

The main guidelines followed by health care providers in our study were the Eating Well with Canada's Food Guide [19] and the Society of Gynecologists of Canada guidelines [20]. Health care providers also mentioned Health Canada's website as a resource. These guidelines, while informative about healthy nutrition, are not sufficient to provide detailed recommendations to women about dietary intakes of calories, cultural foods and food sensitivities.
The Canada Food Guide. I mean just looking at what their healthy diet should be made up of and also there are guidelines put out by them, I think SOGC gives you details about giving different BMIs. So every visit we calculate a woman's weight in kilograms and height so you can calculate their BMI and where they're at. And usually it's their prepregnancy BMI or as close to pre-pregnancy as you can get and then based on their BMI you can advise them how much or how little weight they should be gaining. (HCP FG 1)

Suggestions for exercise in pregnancy were not typically discussed during the prenatal visit. Recommendations were based on the health care provider's belief about a woman's access, or lack of access, to exercise equipment or classes. Assumptions were made about patients' motivation to exercise, so the topic was not featured in prenatal discussions.

The odd time I have recommended doing yoga or aqua yoga or whatever but the thing with that is that it costs money and it's not always cheap. Just a very limited client I think and they can't really afford that kind of stuff. It's something we talk about for sure during a physical, like a non-pregnant physical but somehow in pregnancy I don't know it's not something that comes to mind. Is should make it a point to talk more about that. (HCP, FG 2)

I think it would be hard [to exercise]. Usually they work until the end [of the pregnancy], so after working they are tired and then coming home and looking after kids or cooking or whatever. (HCP FG2)

\section{Discussion}

Results from this study support that intention and behaviour are closely linked to motivation and beliefs around risks and benefits, control and societal norms. Women may be more motivated in pregnancy to establish healthy lifestyle choices, supporting that pregnancy is a unique "teachable moment" [21]. In particular, women are more aware of their health behaviours and needs for their growing baby. Attitude and intention are documented to be strong predictors of exercise behaviour in pregnancy [22]. While nutrition behaviours vary, evidence exists that preconception and pregnancy could indeed be a life event leading to increased general nutrition awareness that might influence women's future nutrition-related behaviours [23]. In some communities, however, financial security is a major barrier to access to healthy food. The women in this study were generally aware of the importance of following nutritional guidelines, and they cited Eating Well with Canada's Food Guide [19] as their primary source of information. They accessed additional information from public health resources when possible, such as community talks and food vouchers. Family commitments, such as older children at home, housework, job responsibilities and health (e.g., nausea) seemed to impact the women's ability to exercise. Of all the exercise that could be performed, walking was the most feasible and least likely to change if it was part of a daily routine (e.g., walking to the bus). A study on predictors of change in physical activity during and after pregnancy also found that women reported decreases in moderate and vigorous physical activity during pregnancy that persisted at 6 months postpartum, however levels of walking did not decline [24].

Health care providers unanimously agreed that healthy weight management during pregnancy is important and indicated that they provided initial guidance on target weight gain for the pregnancy based on maternal BMI at the first prenatal visit. However, in the face of competing priorities in medical issues and lack of time, they found it difficult to address gestational weight management with patients at subsequent visits. Health care providers did not prioritize gestational 
weight gain in terms of risk in relation to other health concerns. They also reported a lack of knowledge in terms of dealing with nutritional counselling and in our sample; physicians did not refer their patients for dietetic advice or specialty assessment unless a more serious health concern arose (such as diabetes). Interestingly, health care providers in this study did not mention any attempts to change this lack of knowledge or consideration of re-prioritizing this issue. There seems to be a focus on symptom and disease management with the current health care system model as a priority over prevention. From the discussions, health care providers agreed that if there were a more definitive prescription of what they should advise women, they would find that clinically useful given their lack of knowledge and time limitations.

Evidence exists that a high proportion of women report receiving incorrect weight gain advice, incorrect or no advice at all about target weight from health care providers in pregnancy $[15,25]$. The present results support that detailed advice on weight management is uncommon and is given primarily to those who are already obese, are middle-aged, or who have comorbidities [26]. Practitioners may be missing important opportunities to counsel mildly overweight individuals to lose weight or to maintain their weight and thereby prevent comorbidities. In previous research, advice and target weight gains were associated strongly with actual weight gain and not receiving advice was associated with weight gain outside of the recommendations [27]. New approaches to provider education and patient communication are needed to implement the IOM guidelines for gestational weight gain [3]. Inter-professional collaboration with other health providers (such as dieticians) would also enable better patient support for prevention measures.

With regards to the feasibility of the planned nutrition and exercise interventions, both were acceptable to pregnant women. Detailed guidance and practical help obtaining specific foods and food intake forms were not considered cumbersome and were understood by women including those for whom English was a second language. Walking seemed feasible for women during pregnancy and not overly demanding, especially if gradually increased. Measurement devices such as pedometers which provide feedback to participants were thought to be motivating. This type of specific feedback, from a device measuring the amount of exercise, could assist women in staying motivated and might be an enabler to lifestyle change as found in other research with pedometers [28,29].

Language barriers and pregnancy complications were the major anticipated barriers to participation in the planned randomized controlled trial.

In conclusion, findings in this study suggest that a clear understanding of risks and benefits, an ability to control and achieve healthy lifestyle choices and support from family/friends and health care providers can improve the likelihood of behavioural change during pregnancy. Pregnant women seem to be a motivated population; however they also face physical, emotional and financial constraints to making lifestyle changes. During pregnancy, health care providers have more frequent visits with women than at other stages of life, thus providing an opportunity to educate and support pregnant women in achieving healthy weight gain during pregnancy. Improved interprofessional collaboration, additional educational resources for health providers and more specific instructions on lifestyle recommendations during pregnancy are needed. Findings from participant women and health care providers in this qualitative study support the acceptability of the interventions planned for the clinical intervention trial.

\section{Acknowledgement}

This research was supported by a Development Grant from the Institute of Human Development, Child and Youth Health of the Canadian Institutes of Health Research; and an operating grant from the Dairy Farmers of Canada. Rishma Walji's post-doctoral fellowship was supported by the Primary Health Care System (PHCS) Program Research Fellowship Award and an operating grant from the McMaster Arts Research Board. We are grateful to the mothers and health care providers who generously volunteered their time and interest in participating in the focus groups.

\section{References}

1. Torrance GM, Hooper MD, Reeder BA (2002) Trends in overweight and obesity among adults in Canada (1970-1992): evidence from national surveys using measured height and weight. Int J Obes Relat Metab Disord 26: 797-804.

2. Statistics Canada. Body composition of Canadian adult 2007-2009. Ottawa, ON: Statistics Canada; 2010. Available at:http://www.statscan.gc.ca/pub/82625-x/2010001/article/11091-eng.htm. Accessed May 24, 2013

3. Institute of Medicine (2009) Re-examination of IOM Pregnancy Weight Guidelines. National Academies Press.

4. Lowell H, Miller DC (2010) Weight gain during pregnancy: adherence to Health Canada's guidelines. Health Rep 21: 31-36.

5. Begum F, Colman I, Mc Cargar LJ, Bell RC (2012) Gestational weight gain and early postpartum weight retention in a prospective cohort of Alberta women. $J$ Obstet Gynaecol Can 34: 637-647.

6. Oken E, Kleinman KP, Belfort MB, Hammitt JK, Gillman MW (2009) Associations of gestational weight gain with short- and longer-term maternal and child health outcomes. Am J Epidemiol 170: 173-180.

7. Vasudevan C, Renfrew M, McGuire W (2011) Fetal and perinatal consequences of maternal obesity. Arch Dis Child Fetal Neonatal Ed 96: F378-382.

8. Gardner B, Wardle J, Poston L, Croker H (2011) Changing diet and physical activity to reduce gestational weight gain: a meta-analysis. Obes Rev 12: e602 620.

9. Thangaratinam S, Rogozinska E, Jolly K, Glinkowski S, Roseboom T, et al. (2012) Effects of interventions in pregnancy on maternal weight and obstetric outcomes: meta-analysis of randomised evidence. BMJ 344: e2088.

10. Oteng-Ntim E, Varma R, Croker H, Poston L, Doyle P (2012) Lifestyle interventions for overweight and obese pregnant women to improve pregnancy outcome: systematic review and meta-analysis. BMC Medicine 10: 47

11. Ajzen I (1985) From intentions to actions: A theory of planned behavior. In: Kuh $\mathrm{J}$ and Beckman J (eds) Action-control: From cognition to behavior. Heidelberg: Springer, 11-39.

12. Azjen I (1971) Attitudinal vs. normative messages: An investigation of the differential effects of persuasive communications on behavior. Sociometry 34 263-280.

13. Ferrari RM, Siega-Riz AM (2013) Provider advice about pregnancy weight gain and adequacy of weight gain. Matern Child Health J 17: 256-264.

14. Willcox JC, Campbell KJ, van der Pligt P, Hoban E, Pidd D, et al. (2012) Excess gestational weight gain: an exploration of midwives' views and practice. BMC Pregnancy Childbirth 12: 102

15. McDonald SD, Pullenayegum E, Bracken $\mathrm{K}$, Chen AM, McDonald $\mathrm{H}$, et al. (2012) Comparison of midwifery, family medicine, and obstetric patients understanding of weight gain during pregnancy: a minority of women report correct counselling. J Obstet Gynaecol Can 34: 129-135.

16. Creswell JW (1998) Qualitative Inquiry and Research Design. Choosing Among Five Traditions, Thousand Oaks, CA: Sage Publications, Inc.

17. QSR International Pty Ltd. (2010) N Vivo qualitative data analysis software Version 9

18. Morse JM, Field PA (1995) Qualitative Research methods for Health Professionals, London: Sage.

19. Health Canada (2007) Eating well with Canada's Food Guide.

20. Davies GA, Maxwell C, McLeod L, Gagnon R, Basso M, et al. (2010) Obesity in Pregnancy. J Obstet Gynaecol Can 32: 165-173

21. Phelan S (2010) Pregnancy: a "teachable moment" for weight control and obesity prevention. Am J Obstet Gynecol 202: 135. 
Citation: Walji R, Wahoush O, Atkinson SA (2013) Feasibility and Acceptance of a Novel Nutrition and Exercise Intervention to Manage Excess Gestational Weight Gain: Focus group study in Ontario, Canada. Primary Health Care 3: 134. doi:10.4172/2167-1079.1000134

22. Downs DS, Hausenblas HA (2003) Exercising for two: examining pregnant women's second trimester exercise intention and behavior using the framework of the theory of planned behavior. Women's Health Issues 13: 222-228.

23. Szwajcer EM, Hiddink GJ, Koelen MA, van Woerkum CM (2007) Nutrition awareness and pregnancy: implications for the life course perspective. Eur $J$ Obstet Gynecol ReprodBiol 135: 58-64.

24. Pereira MA, Rifas-Shiman SL, Kleinman KP, Rich-Edwards JW, Peterson KE, et al. (2007) Predictors of change in physical activity during and after pregnancy: Project Viva. Am J Prev Med 32: 312-319.

25. Stotland NE, Haas JS, Brawarsky P, Jackson RA, Fuentes-Afflick E, et al. (2005) Body mass index, provider advice, and target gestational weight gain. Obstet Gynecol 105: 633-638.
26. Sciamanna CN, Tate DF, Lang W, Wing RR (2000) Who reports receiving advice to lose weight? Results from a multistate survey. Arch Intern Med 160: 2334-2339.

27. Cogswell ME, Scanlon KS, Fein SB, Schieve LA (1999) Medically advised mother's personal target, and actual weight gain during pregnancy. Obstet Gynecol 94: 616-622.

28. Pal S, Cheng C, Egger G, Binns C, Donovan R (2009) Using pedometers to increase physical activity in overweight and obese women: a pilot study. BMC Public Health 9: 309

29. Mottola MF (2009) Exercise prescription for overweight and obese women: pregnancy and postpartum. Obstet Gynecol Clin North Am 36: 301-316. 